\title{
El uso de teléfonos móviles en el sistema educativo público de El Salvador: ¿Recurso didáctico o distractor pedagógico?
}

The use of mobile phones in the public education system in El Salvador: Educational Resource or educational distraction? 



\title{
Reflexion \\ Reality and Reflection \\ año 14, N 40, San Salvador, El Salvador, Centroamérica Revista Semestral Julio-Diciembre 2014 \\ Year 14, N40, San Salvador, El Salvador, Central America Semestral Journal July-December 2014 \\ El uso de teléfonos móviles en el sistema educativo público de El Salvador: ¿Recurso didáctico o distractor pedagógico?
}

\author{
The use of mobile phones in the public education system in El \\ Salvador: Educational Resource or educational distraction?
}

\author{
Herberth Alexander Oliva \\ Bachelor of Education Southern, Texas University \\ Maestro en Administración de la Educación, \\ Universidad Pedagógica de El Salvador. \\ Investigador para el área educativa en el Instituto de Ciencia, \\ Tecnología y Educación de la Universidad Francisco Gavidia. \\ holiva@ufg.edu.sv
}

\section{RESUMEN.}

Este artículo representa la sistematización teórica del estudio titulado El uso de teléfonos móviles en el sistema educativo público: ¿Recurso didáctico o distractor pedagógico? Dicho artículo permite el abordaje pedagógico sobre el impacto que tiene el uso de estos dispositivos en los contextos escolares del sistema educativo salvadoreño. El estudio se realizó en 69 instituciones educativas de los 14 departamentos de El Salvador, generando con ello un marco de análisis en el que el celular parece ocupar el papel de gran distractor del proceso de enseñanza y aprendizaje. El estudio se realizó entre del 6 al 23 de mayo de 2014, tiempo en el que se encuestó a mil 384 estudiantes de tercer ciclo y bachillerato y 200 docentes de distintos puntos del país.

El estudio parte de una relación en el que la dicotomía del uso del teléfono celular en la escuela salvadoreña, permite valorar si dicho artefacto tecnológico es en realidad un recurso didáctico de uso pedagógico o un mero distractor que pone al descubierto diversas tensiones o problemas de la relación existente entre docentes y estudiantes debido a la aparición de este aparato; todo ello en el marco de la postmodernidad pedagógica, en la que el teléfono celular adquiere un gran protagonismo adentro de las aulas salvadoreñas. TECNOLOGÍA EDUCATIVA, INNOVACIONES EDUCATIVAS, MÉTODOS DE ENSEÑANZA. 


\begin{abstract}
This article represents the theoretical systematization of the study entitled The use of mobile phones in the public education system: Educational Resource or teaching resource distractor? This article allows the pedagogical approach on the impact that the use of these devices in Salvadoran educational system contexts. The study was conducted in 69 educational institutions of the 14 departments of El Salvador, thereby generating an analytical framework in which the movile appears to occupy the role of great distractor of teaching and learning. The study was conducted from 6 to 23 May 2014, at which time a thousand 384 junior and high school students and 200 high school teachers from across the country were surveyed. The study is a relationship in which the dichotomy of cell phone use in Salvadoran schools, can assess whether this technological artifact is actually a teaching resource for educational use or a mere distraction that reveals several tensions or problems relationship between teachers and students because of the appearance of this device; all within the framework of educational postmodernism, in which the cell phone takes a leading role in the Salvadoran classrooms. 1. EDUCATIONAL TECHNOLOGY, EDUCATIONAL INNOVATIONS, TEACHING METHODS.
\end{abstract}

\section{Presentación.}

El estudio titulado Uso de teléfonos móviles en el sistema educativo público: ¿Recurso didáctico o distractor pedagógico? es sin duda alguna un acercamiento antropológico y etnográfico al reconocimiento del teléfono celular dentro del aula de la escuela salvadoreña; motivo por el cual el presente estudio debe interpretarse como parte del aporte investigativo y metodológico, que se representa en los diversos estudios académicos que conforman la agenda permanente de proyectos de investigación del Instituto de Ciencia, Tecnología e Innovación de la Universidad Francisco Gavidia (ICTI-UFG).

El marco estructural metodológico que a continuación se describe, se ha extraído del informe preliminar del Centro de Estudios Ciudadanos de la Universidad Francisco Gavidia, dirigido por la licenciada María Isabel Quintanilla de Campos.

\subsection{Generalidades del estudio}

El Centro de Estudios Ciudadanos de la Universidad Francisco Gavidia ha realizado una investigación de opinión pública donde se explora el uso de teléfonos móviles en el sistema educativo público. Para el estudio se utilizó muestreo sistemático probabilístico con afijación proporcional al tamaño de la población de centros educativos por departamento, con un error muestral de más o menos $2.9 \%$. La muestra seleccionada fueron 69 centros educativos de los 14 departamentos del país.

Las encuestas válidas fueron 1,723; entre los entrevistados se tienen 1,541 estudiantes ( $47 \%$ de bachillerato y $53 \%$ de tercer ciclo); 182 encuestas se lograron de los docentes de primero y segundo ciclos.

\subsection{Antecedentes de la investigación}

La línea de investigación "Informática social" parte de los trabajos de Bob Kling "What is Social Informatics and Why Does it Matter?" (1999) y de aplicaciones educativas realizadas por Noriko Hara y Oscar Picardo (Universitat Oberta de Catalunya, 2003).

Básicamente se estudia y analiza desde la perspectiva sociológica y educativa el impacto de las tecnologías de la información y las comunicaciones (TIC's) en la sociedad y en los sistemas educativos. 


\subsection{El auge de la telefonía móvil}

La evolución exponencial de la telefonía celular en El Salvador, según la SIGET, indica que en 2012 teníamos 6.6 celulares por hogar, es decir 8.6 millones de celulares, sobre la base de 5.7 millones de ciudadanos; además, la Encuesta de Hogares de Propósitos Múltiples 2012 señala que los salvadoreños gastan en promedio US\$ 10,8 al mes de telefonía móvil. En el año 2000 había 12.52 celulares por cada 100 habitantes, en 2012 hay 138 celulares por cada 100 salvadoreños.

¿Qué dicen las estadísticas de la UITONU?: 2008, 6.9 millones de suscripciones a celulares; 2009, 7.5 millones; 2010, 7.7 millones; 2011, 8.3 millones; y 2012, 8.6 millones (http://www.itu.int/en/ITU-D/ Statistics/Pages/stat/default.aspx).

\subsection{Preguntas de investigación}

¿Cuánto ha crecido el uso del celular en el sistema educativo público?

¿Existe una correlación entre los programas sociales -subsidios de paquetes escolares- y el crecimiento de celulares? ¿Son los celulares un distractor pedagógico o un recurso didáctico?

\subsection{Aspectos metodológicos.}

Previo al estudio cuantitativo, se realizaron al menos 15 visitas y observaciones cualitativas que permitieron construir un marco hipotético sólido.

Para el muestreo se consideró la Base de Centros Escolares que reportó el Censo
Inicial 2012 del MINED. Desagregado por Nivel Educativo, Grado y Sexo.

Este estudio no tiene precedentes, y debe ser de interés del Ministerio de Educación, ya sea para conocer lo que está sucediendo y así diseñar o ajustar sus políticas y programas.

A pesar de que la ejecución de este tipo de investigaciones educativas son de gran interés para el desarrollo pedagógico de El Salvador, el lunes 5 de mayo de 2014 se envió una nota a la Dirección Nacional de Gestión Departamental del MINED para solicitar su apoyo e involucramiento y nunca contestaron; de tal modo que el estudio se realizó con la colaboración voluntaria de algunos directores, docentes y estudiantes, dentro y fuera de las escuelas, logrando cubrir la muestra representativa diseñada.

\section{Introducción}

Dentro del complejo entramado social salvadoreño, es fácil encontrar fenómenos sociales dignos de un análisis científico, fenómenos que ponen de manifiesto las incongruencias que se viven día a día dentro del aparataje de la escuela pública salvadoreña.Unodelosmúltiplesfenómenos que merecen la atención del Instituto de Ciencia, Tecnología e Innovación de la UFG es el aumento constante de telefonía celular en las escuelas salvadoreñas.

Un ejemplo que evidencia la argumentación sobre la necesidad de la presente investigación, radica en concebir que en el mundo moderno el elemento tecnológico de hoy se vuelve omnipresente en gran parte de la cotidianeidad salvadoreña, en donde... "es muy necesario tener 
algún dominio básico de tecnologías e informática para sobrevivir a los diversos avatares, postmodernistas; uno de esos avatares, son los teléfonos celulares, los cuales se han convertido en parte integral de la vida diaria". ${ }^{1}$ Las personas de todas las edades confían en ellos para la comunicación y el entretenimiento.

Sin embargo, algunas personas no etán de acuerdo en que si los teléfonos móviles son adecuados para ciertos lugares, como el salón de clases. Es importante que los padres de familia, maestros y estudiantes entiendan los efectos nocivos de los teléfonos celulares en las escuelas, argumento más que valedero como para emprender la titánica labor de investigación sobre el fenómeno antes mencionado.

Surge entonces la interrogante ¿Cuál es la importancia de elaborar una investigación que aborde la temática del uso de la telefonía celular en el sistema educativo público salvadoreño?

Más allá de las implicaciones económicas que tiene el uso de la telefonía celular en jóvenes en edades escolares, es imperante argumentar que los estudiantes siempre están bajo una presión académica que los obliga a desempeñar un buen actuar académico y conductual en la escuela; como resultado directo de esta presión el estudiante podría de manera ocasional hacer trampa en una prueba, tomar fotografías inapropiadas durante las actividades escolares o convertir los recursos tecnológicos en elementos distractores de la clase; por lo tanto, se argumenta que los teléfonos móviles son capaces de algo más que llamar y enviar mensajes, los estudiantes pueden acceder fácilmente a Internet, generar acoso cibernético como un elemento derivado de la violencia escolar o, peor aún, cuando se usa el teléfono celular como medio de comunicación entre estudiantes para cometer un hecho delictivo que contraviene el orden social dentro del recinto escolar.

La importancia de una investigación sobre el uso de celulares en la escuela salvadoreña, radica en que dicha investigación intentará demostrar que... "Los estudiantes que usan teléfonos celulares en el recinto escolar, no pueden concentrarse en la lección si están ocupados en el envío de mensajes en sus teléfonos. Por lo que, dicho comportamiento afecta negativamente el ambiente de la clase; además, distrae a los profesores y estudiantes que están tratando de concentrarse en la lección". ${ }^{2} \mathrm{Si}$ un estudiante se centra en los mensajes de texto, es incapaz de absorber la información que está siendo demostrada por el docente en el momento de la clase.

Desde otra panorámica social se puede argumentar que otro elemento derivado del uso de teléfonos celulares en la escuela, está fuertemente vinculado con la violencia escolar, específicamente en el acoso cibernético, el cual se manifiesta cuando los estudiantes han usado sus teléfonos para avergonzar a sus compañeros o maestros. Por ejemplo, pueden grabar las peleas escolares y publicarlas en Internet, además los estudiantes pueden grabar a sus maestros perdiendo los estribos y luego lo publican en sitios como YouTube. El sentido común dictaría que estos actos pueden ser humillantes para las víctimas y perjudiciales para la reputación de la escuela.

Como argumentación final se considera imperante intentar desfragmentar esta 
complejidad social propia de la institución educativa moderna, la cual dentro de sus aulas intenta educar; pero a la vez lucha contra un sistema social que busca un desaprendizaje del andamiaje de valores morales, tan necesarios en la acción formativa. Por lo expuesto... "Es necesario señalar que a pesar de que las desventajas de los teléfonos celulares en las escuelas son bastante evidentes, la decisión de regularlos o prohibirlos corresponde a cada escuela"3 Sin embargo algunas instituciones prohíben los teléfonos celulares, y existen otras que se muestran indiferentes a estos, debido a la presión ejercida por los padres, que afirman que los celulares son importantes para la comunicación de emergencia.

Aun y cuando está plenamente comprobado que los teléfonos celulares son considerados una alteración para el diario vivir en la escuela y en el aula, el uso de los aparatos o componentes telemáticos sigue creciendo día a día, contradiciendo a una sociedad que argumenta ser pobre, pero que dispone de dichos dispositivos dentro de los cánones que impone la moda y el consumo; por lo tanto, la acción de hablar por teléfono celular dentro de la escuela con otros compañeros mediante el uso de mensajes de texto es un incordio para educadores que se oponen a que los estudiantes usen teléfonos celulares, por lo que no es difícil observar que los estudiantes continúen utilizando sus teléfonos móviles para hablar, jugar y no prestar atención en clase.

\section{Problemática en contexto}

(Marco teórico y análisis reflexivo).

La conveniencia de la presente investigación trata de responder a la necesidad de los educadores del contexto educativo salvadoreño, sobre cómo entender los crecientes cambios tecnológicos que la sociedad está experimentando, implicando concretamente el uso de la tecnología celular por parte del conglomerado estudiantil en la escuela salvadoreña.

Dentro de esa línea de análisis es importante destacar que el uso y el abuso constantes del teléfono celular en las aulas de la escuela salvadoreña, está creando una influencia negativa en el aprendizaje activo dentro del salón de clases, por lo que es necesario para las autoridades escolares, determinar hasta qué punto es necesario permitir la utilización de estos dispositivos dentro de las instituciones educativas. No obstante, el sistema educativo salvadoreño también está en la obligación de reconocer la urgente necesidad de la innovación tecnológica en cuanto a instrumentos y metodologías para canalizar el aprendizaje significativo en los estudiantes.

En concreto, es relevante para la sociedad salvadoreña en general la elaboración de este tipo de investigación científica, en la cual sea posible comprobar que si bien es cierto que las tecnologías ayudan a canalizar más y mejor información académica dentro de sus procesos formativos, también es cierto que el mal uso que se le da al teléfono celular como recurso tecnológico en el aula, se ha convertido hoy en día en la problemática más preocupante por resolver dentro del salón de clases; en primera instancia, porque esta herramienta tecnológica está afectando directamente el aprendizaje del educando, y en segunda instancia porque dicha problemática está causando dispersión y afectando negativamente la capacidad de analizar y reflexionar el conocimiento impartido por el conglomerado docente. 


\subsection{El teléfono celular en el aula salvadoreña}

Para contextualizar el aparecimiento del teléfono celular en la sociedad salvadoreña, es necesario remontarse a los primeros años de la década de los 90, años en los que se introduce el teléfono celular al país, gracias a la empresa TELEMOVIL. El auge por el uso de este tipo de tecnología ha sido tan grande, que para opinión del sociólogo salvadoreño Oscar Felipe Shultz existen al menos 2 celulares por cada habitante en el país, lo cual marca una clara complejidad demográfica en un país en el que existen más teléfonos que habitantes.

"El celular dentro de las instituciones educativas adquiere la connotación de ser un símbolo de esta época en lo referente al estatus o poder adquisitivo del estudiante, dando pautas concretas que llevan al análisis en el que dicho objeto se vuelve omnipresente en todas las instituciones educativas, sean estas públicas o privadas". ${ }^{4}$

En el caso de la tenencia del teléfono celular en los estudiantes, la sociedad salvadoreña se enfrenta a la propia dinámica del modelo consumista, en la que portar un teléfono celular es portar un objeto constituido en el simbolismo tecnológico que marca un nuevo latido en la sociedad, que conjuga velocidad y comunicación en cualquier rincón de la sociedad del conocimiento.

La escuela salvadoreña se encuentra invadida por la aparición del teléfono celular, en donde este artilugio electrónico parece ocupar el lugar de la gran interrupción de las clases, las explicaciones y también las evaluaciones que imparte el docente. Dicha situación amerita la elaboración de ciertas reglas que regulen su uso, para lograr una mejor convivencia escolar.
Desde ese sentido, la presente investigación pone al descubierto diversas tensiones $\mathrm{o}$ problemas de la relación pedagógica entre docentes y estudiantes; sin embargo, es necesario reconocer la complejidad que tiene el sostener una clase con adolescentes en esta postmodernidad pedagógica, por lo que se vuelve imperante regular el uso del celular acordando pautas que se ajusten a cada contexto, siempre sujetas a negociaciones futuras. Pero también hay una oportunidad de ver el teléfono celular como acceso a nuevos sujetos sociales en la escuela y a otras conjeturas pedagógicas de las que puedan surgir nuevos recursos para la enseñanza y el aprendizaje, entre muchos otros.

En opinión del célebre pedagogo Dr. Oscar Picardo, el auge del teléfono celular en la escuela salvadoreña puede ubicarse en el año 2009, año en el que de conformidad a políticas gubernamentales, se inicia el "Programa de Paquetes Escolares".

En el artículo titulado... Una peligrosa tesis educativa" el Dr. Picardo señala: "Los paquetes escolares, útiles y uniformes son una medida que surgió en el marco del plan anticrisis de este Gobierno (Gobierno de Mauricio Funes, 2009-2014), con la posible finalidad de reactivar la economía de micros y pequeños empresarios y de garantizar que los niños y las niñas no abandonaran la escuela por razones económicas. Esta medida muy popular y bien evaluada por la ciudadanía la critiqué técnicamente en su momento por dos razones:

a. "No existía un estudio serio de costobeneficio o tasa de retorno que fundamentara su justificación; y

b. A pesar de que la mayoría de niños que están en escuelas públicas provienen de 
hogares de escasos recursos, no todos la necesitaban".

Para el célebre especialista en temas educativos la ejecución de dicho programa, da paso a la formulación de una peligrosa hipótesis educativa, en la cual a partir de un trabajo investigativo con varias escuelas urbanas del Gran San Salvador, se ha detectado un crecimiento abrupto de uso de celulares por parte de los estudiantes; en una primera aproximación -aún no científicaPicardo señalo que 8 de cada 10 niños poseen celulares, de los que 7 de cada 10 casos son "smartphones" (de primer grado a bachillerato); en donde es muy posible que exista una fuerte correlación entre la entrega de uniformes, zapatos y útiles y el crecimiento de celulares en la escuela. La conclusión que Picardo propone es que el Gobierno subsidia 80 millones al año a las compañías Claro, Digicel, Tigo y Movistar, es decir lo que la familia se ahorra en gastar en útiles, zapatos y uniformes se los gastan en teléfonos y saldo (saldo para teléfonos celulares que en muchas ocasiones es vendido en las tiendas de estas escuelas).

Para finalizar su argumento, el Dr. Picardo indica que la evolución exponencial de la telefonía celular en El Salvador, según la SIGET, permite estimar que en el año 2012 El Salvador tenía 6.6 celulares por hogar, es decir 8.6 millones de celulares, sobre la base de 5.7 millones de ciudadanos; además, la Encuesta de Hogares de Propósitos Múltiples 2012 señala que los salvadoreños gastan en promedio US\$ 10,8 al mes de telefonía móvil. En el año 2000 había 12.52 celulares por cada 100 habitantes, en 2012 hay 138 celulares por cada 100 salvadoreños.

\section{El teléfono celular como problema pedagógico en las instituciones escolares salvadoreñas}

A raíz de una serie de situaciones derivadas de los hechos de violencia en que se enmarca a El Salvador como el cuarto país más violento del mundo ${ }^{6}$ (según datos revelados por la ONU), el fenómeno de violencia escolar susitado en diversos planteles educativos, muestra, por parte de algunos estudiantes, el mal uso y hasta con fines delictivos la tendencia que teléfono celular en la institución educativa. En el articulado jurídico que sustenta el actuar educativo nacional es imposible encontrar algún lineamiento que permita establecer criterios consensuados para determinar el buen manejo de los teléfonos. Esto se debe a que simplemente no existen regulaciones al uso de estas tecnologías en el aula de la escuela salvadoreña.

No obstante, hay argumentos que determinan que los celulares representan un elemento distractor y perturbador para la educación. Por lo que será necesario determinar si este medio se puede dejar fuera de los salones de clase, o si estos permanecen apagados durante el tiempo en que los alumnos se encuentren dentro del salón y el plantel. Sin embargo, la situación que constituye el eje central de la presente investigación, es una responsabilidad compartida entre padres de familia que optan por adquirir estos medios de comunicación para sus hijos, y las autoridades escolares que no regulan el uso y manejo de dicha tecnología; por lo que es recomendable una supervisión desde los hogares, para que los estudiantes no se vean inmiscuidos en situaciones bochornosas. 
El uso del teléfono celular en el aula, ante el beligerante clima de violencia que envuelve al sistema educativo salvadoreño, debería estar prohibido; en primer lugar, porque es un elemento perturbador que rompe con los tiempos que debe tener el aprendizaje, por lo que tiende a generar en la comunidad estudiantil expectativas contrarias al clima que reinante en la escuela para cumplir con los objetivos pedagógicos.

Aunque prohibir parezca un verbo políticamente incorrecto y pocos se animen a usarlo en educación, se oyen cada vez más voces que reclaman límites. La lógica del buen desempeño docente necesita de un normativo nacional que reglamente el uso del teléfono celular en las escuelas, para que el mismo docente o las autoridades escolares no tengan inconveniente con las familias de los estudiantes, a los que en más de alguna ocasión se les decomisa dicho artilugio tecnológico cuando son sorprendidos usándolo en clase.

\subsection{Desventajas puntuales del uso del teléfono celular en la escuela salvadoreña}

La argumentación central que deriva en la realización de la presente investigación da paso a desfragmentar de manera concreta las desventajas del uso del teléfono celular en la escuela salvadoreña. Ante ello, el docente tiene la obligación profesional de asumir que esta tecnología (el teléfono celular) es un nuevo sujeto de educación, en donde su aceptación o rechazo dentro de las aulas dependerá de la forma en que el estudiante le dé uso a dicho artefacto; esto da paso a sustentar que la escuela sigue siendo la institución que transmite conocimiento; pero no se puede obviar que las redes sociales, los medios tecnológicos, entre otros, son nuevas formas de educación, tanto dentro como fuera de la escuela.

Algunas desventajas atribuidas al uso del teléfono celular en los salones de clase son:

a. Interrupciones en medio de la clase. Comúnmente el teléfono celular de los estudiantes constituye un distractor al momento de impartir una clase. Esto es por el hecho de que ya se ha hecho común que los teléfonos celulares de los estudiantes suenen a media clase, o que se les descubra haciendo uso de alguna de sus diversas funciones. Es por ello que en la mayoría de las instituciones educativas del país se han establecido reglas que prohíben su uso, e incluso su portación cuando se está en el aula. Sin embargo, la dependencia de los jóvenes hacia el teléfono celular no solamente se ve reflejada en los problemas surgidos por el uso del aparato celular en las aulas; esto se debe a que la mala utilización del teléfono también se extiende a la vida diaria.

b. Pérdida de tiempo escolar: El apego de los jóvenes al teléfono celular es tal que sus actividades diarias en tiempo escolar, muchas veces se centran en dicho aparato, lo que hace que las actividades que antes se realizaban como las conversaciones con sus compañeros y profesores se vean suprimidas y reemplazadas por juegos con el móvil, mensajes de texto, etc. La realidad del aula escolar en algunas instituciones educativas ha demostrado que muchos estudiantes al inicio de su interacción con su primer teléfono celular, utilizan el teléfono con moderación, pero enseguida este empieza a consumirles más tiempo del 
que esperaban, dejan a un lado otras actividades de carácter escolar más importantes que las que normalmente llevan a cabo.

c. Graves afecciones por el uso desmedido e irracional del teléfono celular: La tecnología y la necesidad de mantenerse en contacto han propiciado el crecimiento de teléfonos celulares alrededor del mundo. Estos dispositivos pueden proporcionar llamadas de voz, entretenimiento, mensajes de texto, direcciones y acceso web con un sólo clic en un botón.

Sin embargo, la omnipresencia y comodidad de los teléfonos celulares en los recintos escolares del país, tienen desventajas para la salud de los estudiantes, ya que los teléfonos celulares emiten niveles bajos de radiación (radiofrecuencia), en donde grandes cantidades de esta energía pueden calentar y dañar los tejidos corporales, especialmente alrededor de los ojos y testículos, órganos que no tienen suficiente circulación sanguínea para transportar el calor. También existe la preocupación de que los teléfonos celulares puedan causar cáncer, dolores de cabeza, problemas del sueño y pérdida de memoria.

\subsection{El teléfono celular como distractor de la clase y obstáculo para el aprendizaje.}

A pesar de la condición de país, de tercer mundo, El Salvador tiene dentro de su componente social una fuerte dosis de consumo, por lo que no es raro encontrar a una persona que gane salario mínimo, en posesión de un celular de \$500.00; entendiendo que para los parámetros del modelo económico capitalista, la creación e imposición de satisfacciones innecesarias en los ciudadanos es el verdadero motor del aparato económico consumista.

En el contexto salvadoreño es muy común, en la era de la tecnología y de la competitividad, que los niños y los adolescentes tengan un teléfono celular y a la vez exista un abuso en el uso de estos aparatos electrónicos. Si bien, efectivamente son necesarios, su mal uso debiese ser motivo de reflexión y de alerta para los padres, más aún cuando el mal uso se suscita en el salón de clases.

El teléfono celular se ha convertido en un artículo indispensable en la vida de un adulto y cada vez más entre los niños.Y es que no sólo es un medio para recibir y contestar llamadas; ahora los teléfonos inteligentes o smartphones permiten hacer múltiples actividades como navegar por internet, interactuar en las redes sociales, chatear, tomar fotografías, grabar videos, entre otros.

De hecho la gran mayoría del sector estudiantil de los niveles de educación básica y educación media en El Salvador, se ha convertido en un interesante nicho de negocios para los comerciantes de este tipo de tecnología, que ha derivado en que desarrollen productos que captan el interés de los más pequeños, incluso se han asociado a productores de juguetes para lograr este objetivo. Pero, ¿qué pasa cuando estos aparatos llegan a la sala de clases?, ¿'su uso genera algún daño en los niños en el entendido de que tempranamente están accediendo a ellos?, ¿es un símbolo de estatus o de pertenencia en el grupo de interacción?, ¿pueden aportar al desarrollo cognitivo de los pequeños?

A partir de estas dudas, el presente estudio pretende determinar todo un análisis de óptica y perspectiva social, en el que 
se señala que los teléfonos dentro de la escuela salvadoreña han tenido un aumento sustancial, pero no se han previsto los peligros que esto conlleva. Por lo tanto, y bajo un marco reflexivo psicosocial, es válido señalar que entre las principales consecuencias del uso de celular dentro del aula estarían en la creación de ciertas identidades falsas, ya que los estudiantes, con el uso de las redes sociales, crean la configuración de un falso ser, como una falsa identidad en donde podrían llegar hasta tener dos tipos de personalidades: una a través de la red social y otra a través de la socialización directa que se hace con los demás estudiantes.

Además, se debe argumentar que dentro de los procesos de interacción social escolar que se da en el sistema educativo salvadoreño. Es fácil determinar que el excesivo uso de celular puede conllevar falta de atención, falta de concentración, desinterés y desmotivación en el aula y hacia los profesores específicamente, o de cualquier otra actividad que esté relacionada con los procesos formativos escolares.

Lo complejo y difícil del entramado social en el que se desarrolla el problema de convivencia escolar entre los estudiantes y el uso del teléfono celular versus el docente que percibe en la tecnología un elemento distractor del aprendizaje, demuestra la existencia de un desfase entre el avance de la tecnología y el buen uso de la misma. Por un lado, hay una preocupación por usar masivamente los celulares, las computadoras y cualquier otra tecnología de la información y la comunicación; pero, por otra parte, no hay una preparación del buen uso o una preparación para ella, creciendo la problemática de lo que ocurre en el salón de clases y dentro de las familias de dichos estudiantes.
Como resultado directo del aparecimiento del teléfono celular en la escuela salvadoreña, existe una tensión entre los profesores y los estudiantes debido al conocimiento de las tecnologías. Hoy día hay algunos estudiantes, que tienen mucho mejor manejo de las formas y los artilugios electrónicos que los profesores, y eso ha generado tensión en la sala de clases, provocando que los profesores tengan dificultades para enfrentar a los estudiantes de una manera distinta; por lo que cada día es más recurrente la idea de que es un error entregar teléfonos celulares a estudiantes muy jóvenes, porque ello impide darse cuenta de que se va ensimismando al estudiante, logrando que este se vaya encerrando en el mundo tecnológico y vaya perdiendo la capacidad de desarrollar sus habilidades sociales tanto dentro de sus familias como con sus compañeros de clase.

\section{Análisis de los resultados encontrados en la investigación}

- Tipo de estudio: Cuantitativo.

- Técnica de registro: Cuestionario individual pre-estructurado.

- Tipo de entrevista: Visita a centros educativos.

- Informante: Estudiantes (Tercer ciclo, Ed. Media) y docentes (educación básica).

- Trabajo de campo: del 14 al 23 de mayo de 2014.

- Entrevistas efectivas: 1,723 boletas válidas a nivel nacional, proporcional al número de centros educativos de cada departamento.

- Muestreo: Probabilístico con afijación proporcional.

- Nivel de confianza: 95\%, error muestral +- 2.9. 


\subsection{Datos sobre los estudiantes encuestados}

Figura 1. ¿Qué grado estudias actualmente?

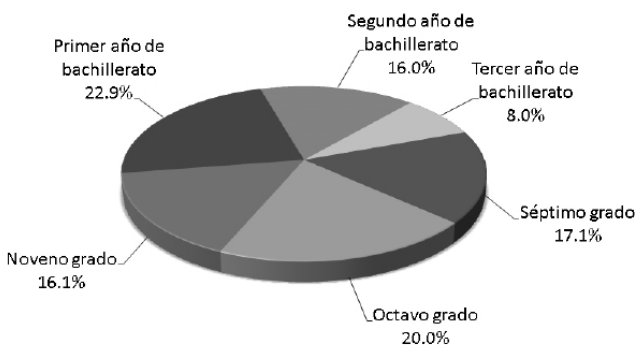

Interpretación. En el análisis inferencial que se puede hacer de la gráfica, se encuentra que la mayor parte de los entrevistados proviene de educación media, en donde cronológicamente enfrentan una adolescencia en la que sus procesos de análisis y reflexión tienden a ser más objetivos y puntuales que los de la etapa de la infancia. Destaca además que en su calidad de adolescentes, tienden a valorizar en mayor medida sus procesos de socialización escolar durante la etapa del bachillerato.

Figura 2. ¿Con quién vives?

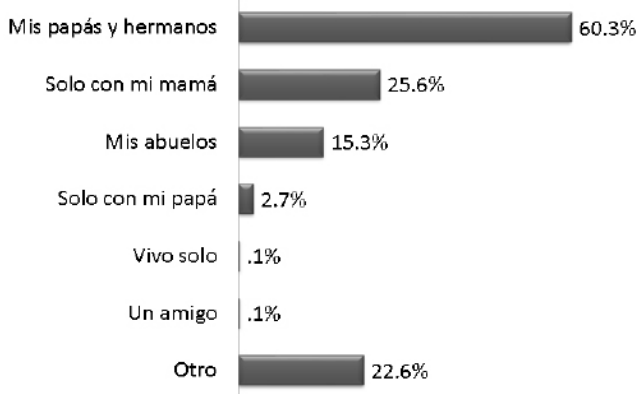

Interpretación. La gráfica refleja que el 60.3 de los estudiantes abordados en el proceso de investigación, vive con ambos padres. Para el caso de la investigación, la convivencia de los estudiantes con ambos padres presupone en alguna medida la concreción de procesos de acompañamiento psicoafectivo a los estudiantes abordados; y en algunos casos, mejores condiciones económicas de vida.

Lo anterior se posiciona como la némesis del problema de desintegración familiar, que en la mayoría de los casos se debe a la irresponsabilidad de uno de los progenitores, o cuando uno o ambos padres de familia buscan emigrar a otras naciones debido al factor socioeconómico salvadoreño, en el que la pobreza y desempleo los obligan a dejar con algún familiar a sus hijos.

\section{Figura 3. ¿Tu familia tiene celular?}

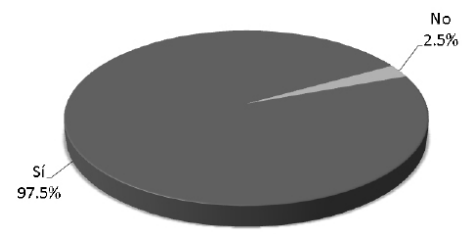

Interpretación. El análisis concluyente, derivado de la presente grafica, denota que el 97.5 de los estudiantes abordados, manifestaron que sus familias poseen teléfonos celulares, por lo que estos, desde muy temprana edad, ven el uso de dichos artilugios como algo normal y cotidiano dentro de sus núcleos familiares, dándoles pauta a la interiorización de la idea de que el uso de los teléfonos en los recintos escolares no debería ser visto como algo malo nicontraproducente para el hecho educativo.

Una de las razones por las que es viable encontrar que la gran mayoría de las familias 
de los entrevistados posean teléfonos celulares, es debido al rol comunicacional que tiene dicho aparato, que en algunos casos se vuelve además un instrumento de diversión y ocio.

Figura 4. Evolución de la telefonía celular ¿Tú tienes celular?

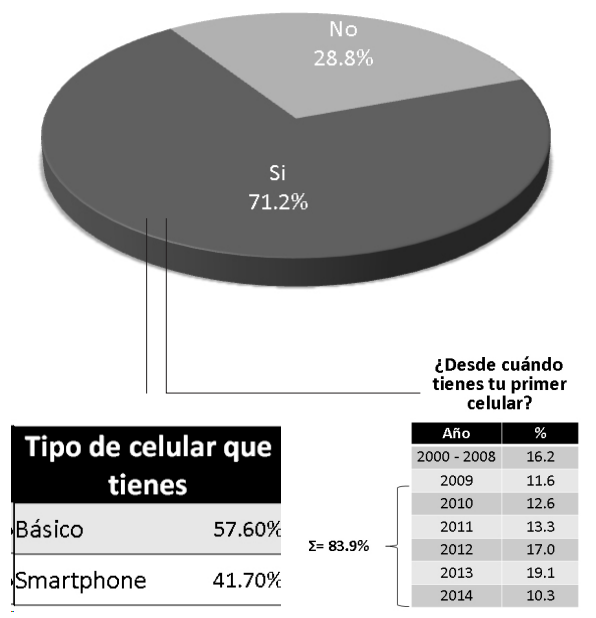

Interpretación. El enfoque de análisis con el que se interpreta la presente gráfica, parte de la idea fundamental de que la mayor parte de entrevistados posee un teléfono tipo básico $57.60 \%$, que suele caracterizarse por no poseer cualidades de navegación a internet, ya que suelen ser de uso exclusivo para emitir o recibir llamadas; sin embargo, un nada despreciable $41.70 \%$ de los encuestados afirma tener un teléfono categorizado como teléfono inteligente, debido a las características particulares, entre las que más destaca su permisibilidad de conexión a internet.

Otro de elementos de análisis que se suma al estudio es el hecho de que al cuestionar a los entrevistados, ¿desde cuándo tienen su primer celular? La mayor parte, constituida en un $19.1 \%$, dijo que su primer teléfono celular fue adquirido en el año 2013, asumiendo el hecho de que el uso extendido a los sectores populares de la sociedad, del teléfono celular, es un fenómeno de reciente data.

Figura 5. ¿Cuántos aparatos tienes?

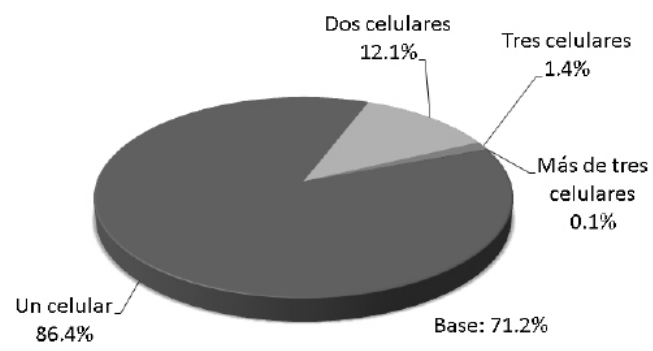

Interpretación. La gran mayoría de los entrevistados, según la grafica, representa que un $86.4 \%$ posee únicamente un teléfono celular; sin embargo, y muy a pesar de que es una minoría, destaca un $1.4 \%$ que sostiene ser poseedor de 3 teléfonos celulares, lo lleva a la conclusión de que entre más teléfonos celulares se posea, mayor será el gasto monetario en saldo y en energía eléctrica al momento de recargar la batería de dichos aparatos.

Figura 6. ¿Cuánto recargas a la semana?

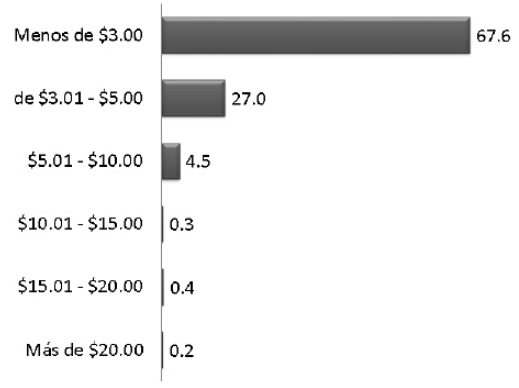


Interpretación. Desde el enfoque financiero que manifiesta la gráfica en estudio, se puede argumentar que la gran mayoría de las personas abordadas en la investigación, deroga un presupuesto de sus economías familiares para la compra de saldo a sus aparatos telefónicos celulares. Los rangos de consumo van en una mayor parte, de menos de \$3.00 a la semana, seguido muy de cerca por aquellos que gastan entre $\$ 3.01$ a $\$ 5.00$ semanales. Cada vez es más notable que el gasto en saldo para teléfonos celulares se vuelve indispensable en los presupuestos familiares, todo ello debido a la dependencia y al uso desmedido de estas tecnologías.

\section{Figura 7. ¿Para qué utilizas el celular?}

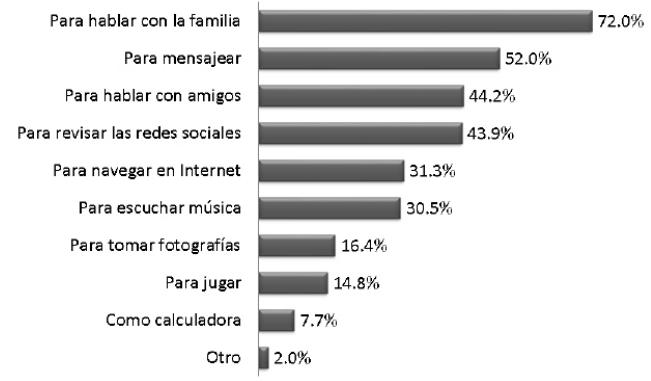

Interpretación. Tal y como se concretizaba en uno de los análisis anteriores, el teléfono celular tiene una utilidad mayormente concentrada en la comunicación familiar; sin embargo, y debido a lo multifacético del componente tecnológico, existe un $52 \%$ de los abordados en el proceso de investigación que señala que el uso que le dan al teléfono celular está en el envío de mensajes. Lo práctico y útil que es el teléfono celular como un medio alternativo de comunicación deriva en su portabilidad y fácil uso y dominio tecnológico que pueden tener esos aparatos; dicho proceso comunicativo distingue la forma de existencia del hombre, por lo que puede entenderse como una forma de relación interpersonal en la actividad humana con los dispositivos electrónicos.

\section{Figura 8. ¿Usas el celular en la escuela o en el instituto?}

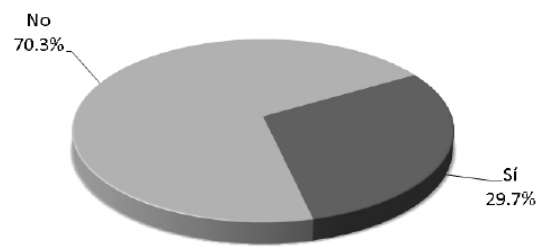

Interpretación. Aun y cuando la población abordada en el proceso de investigación son estudiantes del sector publico educativo, el 70.3\% manifestó que no utilizaba el teléfono celular dentro de los recintos escolares, suscitándose una contradicción entre lo manifestado a nivel general por autoridades escolares y docentes, quienes atribuyen al teléfono celular ser uno de los elementos que repercuten en el fracaso escolar, debido a la gran distracción que estas tecnologías generan. No obstante, en la gráfica se demuestra que existe un valioso $29.7 \%$ que asegura utilizar el teléfono celular en el interior de la institución educativa.

Figura 9. ¿Quién paga tus gastos de celular?

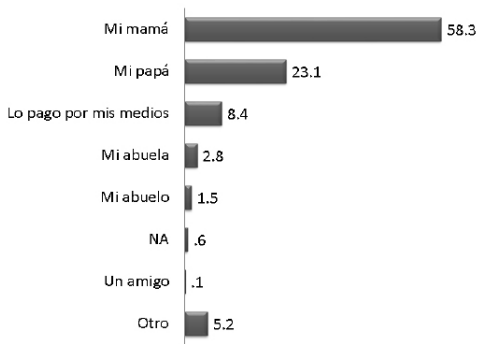


Interpretación. Aun y cuando la población abordada en el proceso de investigación son estudiantes del sector publico educativo, el $70.3 \%$ manifestó que no utilizaba el teléfono celular dentro de los recintos escolares, suscitándose una contradicción entre lo manifestado a nivel general por autoridades escolares y docentes, quienes atribuyen al teléfono celular ser uno de los elementos que repercuten en el fracaso escolar, debido a la gran distracción que estas tecnologías generan. No obstante, en la gráfica se demuestra que existe un valioso $29.7 \%$ que asegura utilizar el teléfono celular en el interior de la institución educativa.

Figura 10. ¿Está prohibido usar el celular en la institución?

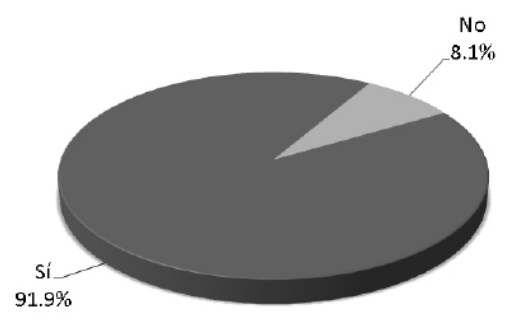

Interpretación. La grafica representa la total y rotunda confirmación por afirmar que dentro de las instituciones escolares existe una prohibición del uso de teléfonos celulares, que marca el 91.9\%. Algunas instituciones retoman esta prohibición como forma frontal de evitar mayores distractores en las clases; pero por otro lado, se encuentra el aspecto de seguridad y privacidad, ya que debido a la facilidad de los teléfonos celulares para tomar fotografías y videos, algunas autoridades escolares tienden a restringir de forma total su uso; no obstante, en el abordaje metodológico de la pregunta, se detectó un $8.1 \%$ de estudiantes que señaló que no era prohibido usar el teléfono celular en sus instituciones educativas

Figura 11. ¿Tus maestros tienen celular?

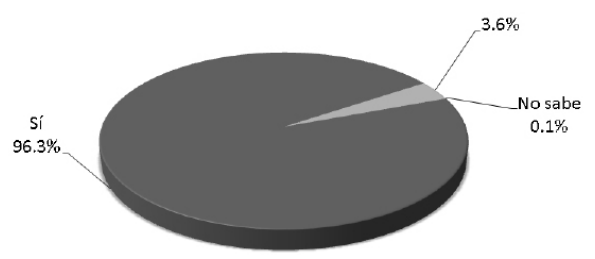

Interpretación. Ante el cuestionamiento a los estudiantes de si conocían si sus docentes tenían teléfonos celulares, se puede visualizar en la gráfica que el $96.3 \%$ de los docentes posee teléfono celular. Lo anterior fundamenta la cotidianidad en el uso y manejo de los teléfonos celulares por parte de las grandes mayorías sociales salvadoreñas.

\section{Figura 12. ¿Tus maestros usan el celular en la clase?}

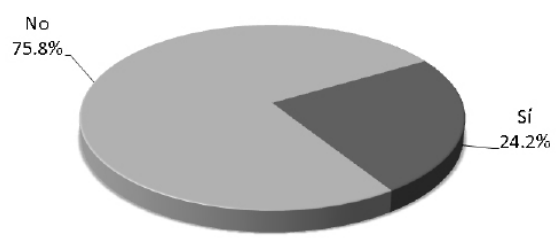

Interpretación. El 75.8\% de los encuestados, argumentó que sus docentes no usaban el teléfono celular en clases; sin embargo, preocupa un destacado $24.2 \%$ que afirma que sus docentes sí usan dichos aparatos durante el transcurso de la clase, lo cual transgrede las normas de conducta y del buen desempeño profesional docente; esto debido en gran parte a que los estudiantes canalizan en mejor forma el aprendizaje que aquellas normas modeladas por sus 
docentes y no por aquellas señaladas en forma teórica.

Figura 13. ¿Eres beneficiario de paquetes escolares, vaso de leche u otro programa social del gobierno?

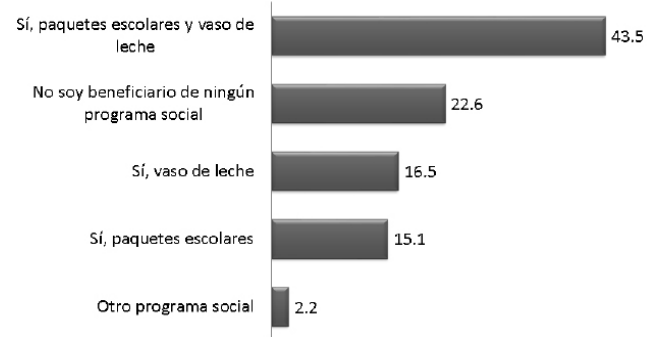

Interpretación. Del total de entrevistados el $71.2 \%$ señala que poseían teléfonos celulares; sin embargo, al profundizar los hallazgos encontrados en la lámina actual, el $43.5 \%$ es beneficiario de paquetes escolares y vaso de leche, interpretando que de cada 7 niños que fueron abordados, 4 tienen teléfonos celulares. Por lo tanto, se puede inferir que el uso de celulares está en alguna forma vinculada con el gasto nulo por parte de algunas familias en lo referente a útiles, uniformes y la merienda escolar. Se disminuye el gasto en insumos escolares, pero aumenta de manera dramática el gasto en telefonía celular.

\section{Figura 14. ¿Sus estudiantes tienen celular en el centro escolar?}

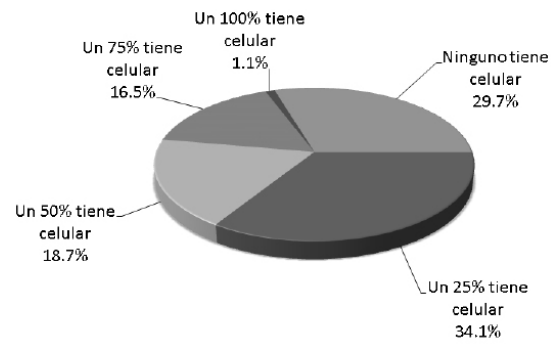

Interpretación. Es importante, a fines aclaratorios, señalar que durante la investigación también se abordaron a algunos docentes, quienes formaron parte de la población sujeta de investigación. A ellos se les cuestionó si sus estudiantes tenían teléfonos celulares, a lo cual la gráfica demuestra que existe una mayoría representada en un $34.1 \%$ que sostiene que un $25 \%$ de sus estudiantes tiene teléfonos celulares. La realidad escolar denota que el uso de dichas tecnologías en el aula es innegable, y cada día preocupa más y más el papel de distractor connotado que adquiere el teléfono celular en la escuela salvadoreña.

Figura 15. ¿Desde cuándo usted ha identificado incremento significativo del uso de celulares en la institución?

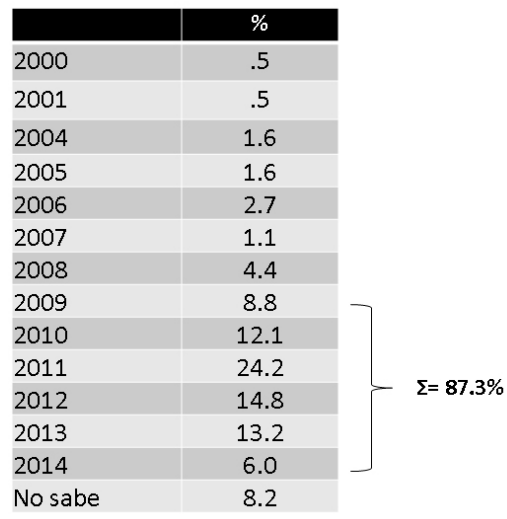

Interpretación. Se le cuestionó a los docentes sobre cuál era el año en el que ellos, percibieron en sus centros educativos un mayor uso de teléfonos celulares por parte de los estudiantes, a lo cual señalaron que fue durante el año 2011, con un $24.2 \%$, mientras que el argumento de los estudiantes fue de que su primer teléfono celular lo adquirieron durante el 2013. Cabe 
mencionar que tanto las fechas que señalan docentes y estudiantes están enmarcadas durante el gobierno de Mauricio Funes, quien fue responsable de la gestión gubernamental sobre la cual se implementó la política de los paquetes escolares gratuitos.

Figura 16. ¿Para qué cree usted que utilizan el celular los estudiantes?

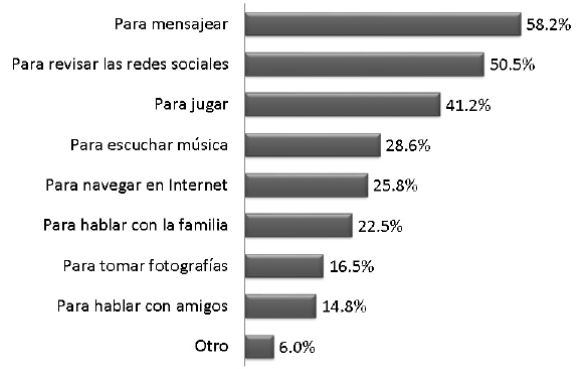

Interpretación. Los docentes señalan, bajo un $58.2 \%$, que los estudiantes de sus centros educativos utilizan el teléfono para enviar y recibir mensajes de texto, esto permite argumentar que los estudiantes perciben en dicho aparato un artefacto para generar procesos de socialización mediante su centralización en las redes sociales, en lugar de utilizarlo como el medio de comunicación para situaciones elementales y emergentes.

Figura 17. ¿Han considerado proponer el uso del celular como un recurso didáctico para los estudiantes?

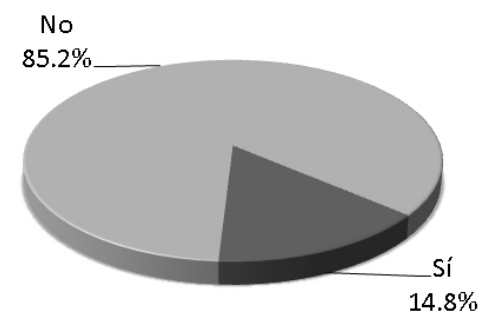

\begin{tabular}{|l|c|}
\hline \multicolumn{1}{|c|}{ Sí, ¿Por qué? } & $\%$ \\
\hline Por el acceso a Internet & 14.8 \\
\hline \multicolumn{1}{|c|}{ No, dPor qué? } & $\%$ \\
\hline Es un distractor & 50.5 \\
\hline Le dan mal uso & 20.3 \\
\hline No tienen edad & 6.0 \\
\hline No tienen recursos económicos & 8.2 \\
\hline
\end{tabular}

Interpretación. Al preguntarle a los docentes si habían considerado proponer el uso del teléfono celular como un recurso didáctico, un $85.2 \%$ manifestó que nunca lo había considerado, respuesta que sin duda alguna pone al ruedo del análisis que gran parte del conglomerado docente está divorciado de las nuevas tendencias metodológicas y desconoce el uso y manejo de las nuevas tecnologías, anteponiendo como pretexto que el teléfono celular es un distractor, obviando de manera categórica el gran potencial de dicho aparato como recurso didáctico bajo determinadas normas académicas.

Figura 18. ¿Qué tanto valoran el uso del celular en el centro escolar, como un recurso de distracción en su proceso de aprendizaje?

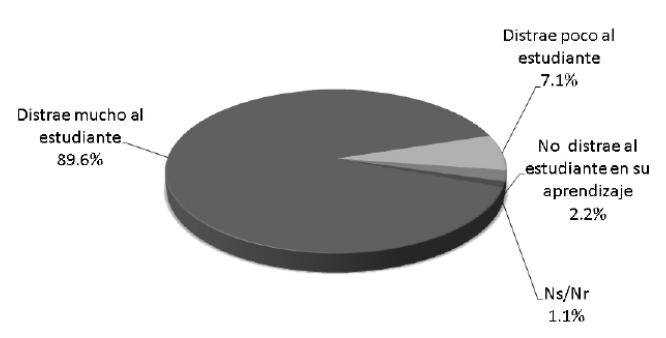

Interpretación. La interpretación de la gráfica conduce a la conclusión de que los docentes consideran que el teléfono celular es verdaderamente un amplio distractor 
académico en los tiempos de clase, motivo por el cual es válido pensar que muchos de los docentes se niegan aun a considerarlo como una herramienta de aprendizaje, debido en gran medida al mal uso que dan los estudiantes a dichos aparatos electrónicos.

\section{Conclusiones}

A modo de conclusión se puede señalar que en los últimos años la tecnología ha tenido grandes avances, y la tecnología específicamente de la telefonía celular no ha sido la excepción, pues esta última se ha ido renovando e innovando según las exigencias de las personas, así como también su rápida y popular expansión principalmente en los jóvenes; sin embargo, esta popularidad no es sinónimo de hacer un buen uso de esa tecnología, pues, según la investigación realizada, se obtuvo que la telefonía celular está afectando el aprendizaje de los jóvenes, ya que estos muestran dispersión, falta de concentración, actitud poco reflexiva y crítica, baja calidad de las actividades, etc., $y$, como consecuencia de esto, un bajo rendimiento escolar.

Al indagar sobre el uso de la tecnología celular por el conglomerado estudiantil y su

\footnotetext{
Notas

1 Prensky Marc (2001). Nativos Digitales, Inmigrantes Digitales. En On the Horizon (MCB University Press, Vol. 9 No. 6, December 2001).

2 A. Estévez-González, C. García-Sánchez , C. Junqué (1997). La atención: una compleja función cerebral. REV NEUROL 1997; 25 (148).

3 Navarro, Miguel (2013). El m-learning y los usos de tablets y celulares en el aula de clase, ¿distractores o dinamizadores del aprendizaje? REVISTA ELECTRÓNICA PRAXIS INVESTIGATIVA ReDIE. Vol. 5, No. 8; Enero-Junio de 2013.
}

influencia en el aprendizaje activo dentro del salón de clases, se obtuvo una tendencia a encauzar al celular como el principal enemigo actual del aprendizaje, pues su inadecuado uso interfiere en el avance escolar, ya que los estudiantes prefieren dar prioridad a la tecnología de vanguardia que tienen en su teléfono celular que a lo que se explica en clases.

Por lo anterior se señalan como conclusiones generales los siguientes apartados:

- Existe una correlación en el crecimiento del uso de celulares con la entrega de paquetes escolares, a partir de 2009.

- Es posible que el clima de violencia permita que los padres de familia les den celulares a sus hijos por razones de seguridad.

- No existe evidencia de propuestas técnicas para utilizar educativamente el celular en el marco de una generación de "nativos digitales".

- Se debe diseñar una estrategia didáctica para integrar el uso de celulares como un medio alfabetizador funcional para tecnologías.

Artículo recibido: 21 de julio de 2014 Artículo aprobado: 25 de octubre de 2014 


\section{Bibliografía.}

A. Estévez-González, C. García-Sánchez , C. Junqué (1997). La atención: una compleja función cerebral. REV NEUROL 1997; 25 (148).

Brown, B. (2002). Studying the use of mobile technology. En B. Brown, N. Green \& R. Harper (Eds.), Wireless world. Social and interactional aspects of the mobile age (pp. 3-15). Londres: Springer-Verlag.

Cabrera, D. H. (2006). Movimiento y conexión. Política y Sociedad, 2, (46), 91-105.

Cooper, G. (2002). The mutable mobile: social theory in the wireless world. En B. Brown, N. Green \& R. Harper (Eds.), Wireless world. Social and interactional aspects of the mobile age (pp. 19-31). Londres: Springer-Verlag.

Cutting the cord. (1999, 9 de octubre). The Economist, pp. 6-12.

De Sola Pool, I. (Ed.). (1977). The social impact of the telephone. Cambridge: MIT Press.
Dimmick, J. W., Skiand, J. \& Patterson, S. J. (1994, octubre). The gratifications of the household telephone. Sociability, instrumentality, and reassurance. Communication Research, 21 (5), 643-663.

Prensky Marc (2001). Nativos Digitales, Inmigrantes Digitales. En On the Horizon (MCB University Press, Vol. 9 No. 6, December 2001).

Engel, G.; Green, T. (2011). Cell Phones in the Classroom: Are We Dialing up Disaster? En: TechTrends: Linking Research and Practice to Improve Learning, v55 n2 p39-45 Mar 2011.

Navarro, Miguel (2013). El m-learning y los usos de tablets y celulares en el aula de clase, :distractores o dinamizadores del aprendizaje? REVISTA ELECTRÓNICA PRAXIS INVESTIGATIVA ReDIE. Vol. 5, No. 8; Enero-Junio de 2013.

Lagos, Ivonne (2011). Percepciones respecto al uso de herramientas tic en el aula: Estudio de caso desde la perspectiva de profesores(as) rurales. Tesis para optar al título de Profesora de Lenguaje y Comunicación. Universidad Austral de Chile. 